\title{
ESTRUTURA DE CUSTOS DO MANEJO DE BAIXO IMPACTO EM FLORESTAS TROPICAIS - UM ESTUDO DE CASO
}

\author{
Romano Timofeiczyk Junior*, Luiz Roberto Graça**, Ricardo Berger***, \\ Roberto Antonio Ticle de Melo e Sousa****, Roberto Tuyoshi Hosokawa***** \\ *Eng. Florestal, Dr., Depto. de Economia Rural e Extensão, UFPR - romano@floresta.ufpr.br \\ **Eng. Agrônomo, Economista, Dr., EMBRAPA-Floresta, - lgraca@cnpf.embrapa.br \\ ***Eng. Florestal, Dr., Depto. de Economia Rural e Extensão, UFPR - berger@floresta.ufpr.br \\ **** Eng. Florestal, Economista, Faculdade de Engenharia Florestal, UFMT - ratms@terra.com.br \\ ***** Eng. Florestal, Dr., Depto. de Ciências Florestais da UFPR - hosokawa@floresta.ufpr.br
}

Recebido para publicação: 13/10/2004 - Aceito para publicação: 30/04/2005

\begin{abstract}
Resumo
Estrutura de custos do manejo de baixo impacto em florestas tropicais - um estudo de caso. Este estudo teve o objetivo de analisar de forma pontual a estrutura de custos do manejo de baixo impacto em florestas tropicais com dois ciclos de corte. Os dados utilizados foram fornecidos por uma empresa que explora uma floresta situada no município de Marcelândia, Estado do Mato Grosso, e comercializa as toras com as indústrias da região. Os resultados demonstraram elevação dos custos variáveis ao longo do primeiro ciclo em função da implantação dos tratos silviculturais, com o custo fixo permanecendo inalterado. No segundo ciclo, ocorreu redução no custo total em razão do aumento da produtividade para $15 \mathrm{~m}^{3} /$ ha e da eliminação de operações das atividades pré-exploratória e exploratória. Dos componentes do custo fixo, o custo administrativo é o mais elevado, representando $26,2 \%$ do custo total, ou $\mathrm{R} \$ 16,27 / \mathrm{m}^{3}$. Dos custos variáveis, a atividade exploratória é a mais onerosa, custando R $\$ 22,33 / \mathrm{m}^{3}$, dos quais $\mathrm{R} \$ 12,92 / \mathrm{m}^{3}$ são consumidos pela abertura de estradas e o arraste. Palavras-chaves: custos do manejo de baixo impacto; floresta tropical; estrutura de custos.
\end{abstract}

\begin{abstract}
Structure of costs for low impact manegement in the tropical forest-a case study. This work had the objective to analyze the structure of the cost of low impact management in the tropical forest in two production cycles. The data utilized were supplied from the company that explores a forest land localized In the Marcelândia city, State of Mato Grosso and commercializes the logs with the industries of the region. The results showed the increasing of the variables costs to long of first cycle in function of the silvicultures treatments, with the same fixed costs. In the second cycle it was observed a reduction in the total cost due to the increasing of productivity to $15 \mathrm{~m}^{3} / \mathrm{ha}$, and the elimination of pre-harvesting and harvesting operations. Inside the fixed cost, the administrative cost is higher, with $26.2 \%$ of the total cost or $\mathrm{R} \$ 16.27 / \mathrm{m}^{3}$. From the variables costs, the harvest activity is the more expensive, having a cost of $\mathrm{R} \$ 22.22 / \mathrm{m}^{3}$, from which $\mathrm{R} \$ 12.92 / \mathrm{m}^{3}$ accounted for road construction and skidding operations.
\end{abstract}

Keywords: low impact management costs; tropical forest; structure of costs.

\section{INTRODUÇÃO}

A Amazônia legal possui 5 milhões de $\mathrm{km}^{2}$, dos quais 4 milhões, ou aproximadamente $80 \%$ são cobertos por densa floresta tropical (Arima; Veríssimo, 2002). As áreas não florestais, como cerrados e campos naturais são de 19\%, enquanto lagos e rios compreendem 1\% da Amazônia. Essa região é uma das mais importantes regiões fitogeográficas do mundo, pertencendo ao domínio da floresta latifoliada perene. Possui um valioso patrimônio genético, representado por milhares de espécies animais e vegetais, além de um ciclo hidrológico da maior bacia hidrográfica do mundo, responsável por $20 \%$ de toda a água que é lançada no oceano, e um subsolo que oculta um vasto patrimônio em riquezas minerais (Zachow, 1999). 
A floresta Amazônica tornou-se alvo das pressões nacionais e internacionais, em função da sua biodiversidade e da importância que tem como reguladora do clima, na proteção de mananciais e dos solos, além de grande fornecedora de produtos madeireiros e não-madeireiros. A necessidade da utilização racional dos recursos naturais confere ao manejo florestal um importante instrumento para compatibilizar o desenvolvimento econômico, social e ambiental.

Entretanto, de acordo com Cunha (2003), na prática é difícil definir o que se entende por manejo florestal sustentável. As interações entre flora e fauna em um ecossistema florestal são complexas e difíceis de entender, haja vista que ações tomadas no presente somente serão mostradas com mais evidências em sucessivos ciclos de corte. Algumas técnicas desenvolvidas apresentam restrições, principalmente em escala comercial. De acordo com Acselrad (1995), para dizer que uma determinada prática florestal é sustentável, é preciso recorrer a uma comparação de atributos entre dois momentos situados no tempo: entre passado e presente, entre presente e futuro. Como as poucas áreas manejadas encontram-se ainda no primeiro ciclo de corte, há dificuldade de determinar se as técnicas atualmente desenvolvidas conduzem à sustentabilidade do manejo florestal.

Apesar da normatização do Plano de Manejo Florestal Sustentado, Hummel (2001) enfatiza que as informações relacionadas a esses planos são escassas e de pouca confiabilidade. As causas, entre outras, são: a) problemas no processamento, sistematização e ordenamento dos dados; b) planos com informações distorcidas e incompletas; c) ausência de dados sobre a área/volume/espécie de exploração anual; d) não execução no campo e; e) informações sobre a área e volume total do plano de manejo revelam pouca coisa.

As pesquisas desenvolvidas para comprovar a viabilidade do manejo florestal madeireiro têm privilegiado principalmente a verificação de aspectos técnico-científicos. As análises econômicas ainda estão limitadas a experiências em projetos demonstrativos, carecendo de informações sobre o comportamento econômico em escala empresarial e envolvendo mais de um ciclo de corte, que é o período decorrido entre dois momentos de exploração na mesma área (Souza, 2002).

Para verificar se determinada prática de manejo florestal é sustentável, é necessária uma grande quantidade de informações que demonstrem o efeito da exploração e da atividade pós-exploratória na regeneração da floresta, o que é difícil, já que as florestas manejadas encontram-se ainda no primeiro ciclo de corte. Portanto, um dos grandes obstáculos para determinar a viabilidade econômica do manejo sustentado de baixo impacto é a falta de informações consistentes. Apesar dessas limitações, este trabalho procurou colaborar com informações sobre a estrutura de custos do manejo de florestas tropicais.

\section{MATERIAL E MÉTODOS}

\section{Área de estudo}

A descrição da fazenda que gerou os dados para a realização desse trabalho foi fornecida pelo Projeto de Apoio ao Manejo Florestal Sustentável na Amazônia (ProManejo) - MMA/IBAMA/PPG7, Projeto Huaia-Missu. A Fazenda está localizada no município de Marcelândia, Estado do Mato Grosso, a $873 \mathrm{~km}$ de Cuiabá e produz toras a partir de floresta tropical, tendo como principal destino da produção as indústrias de laminados e serrados dos municípios de Sinop e Marcelândia, no Mato Grosso.

A área total da Fazenda é de 145.000 ha, e a Área de Manejo Florestal (AMF) é de 116.348 ha. O tamanho de cada Unidade de Produção Anual (UPA), que é a área a ser explorada anualmente, é de 4.653,92 ha.

\section{Fonte de dados primários}

A empresa responsável pela execução do manejo florestal e pela comercialização das toras forneceu os dados de todos os recursos físicos e financeiros necessários para a execução do manejo florestal.

\section{Fonte de dados secundários}

Os dados secundários foram obtidos no Instituto Brasileiro do Meio Ambiente e dos Recursos Naturais Renováveis (IBAMA), Ministério do Meio Ambiente (MMA), Sindicato das Indústrias Madeireiras do Norte do Mato Grosso (SINDUSMAD), e Inflor - Engenharia Florestal. 


\section{Identificação das atividades e operações}

Foram considerados dois ciclos de corte de 25 anos cada um, baseado na Instrução Normativa 04 do Ministério de Meio Ambiente (MMA), que regulamenta o manejo florestal em escala empresarial na região Amazônica. Na Figura 1 está representada a metodologia adotada, onde cada quadrado representa uma UPA, do total de 25 UPAs. O primeiro ciclo de corte pode ser considerado como implantação do sistema de manejo florestal, onde são exploradas as UPAS de número 1 a 25 . Ao iniciar o segundo ciclo de corte, cujo sistema de manejo florestal já está implantado, retorna-se na primeira UPA, representada pela simbologia 1s na Figura 1. Esse procedimento repete-se ao longo dos anos.

Figura 1. Unidades de produção anual (upa) nos dois ciclos de corte.

Figure 1. Annual production units in the two cutting cicles.

\begin{tabular}{|c|c|c|c|c|c|c|c|c|c|}
\hline 1 & 2 & 3 & 4 & 5 & 6 & 7 & 8 & 9 & 10 \\
\hline 11 & 12 & 13 & 14 & 15 & 16 & 17 & 18 & 19 & 20 \\
\hline 21 & 22 & 23 & 24 & 25 & $1 \mathrm{~s}$ & $2 \mathrm{~s}$ & $3 \mathrm{~s}$ & $4 \mathrm{~s}$ & $5 \mathrm{~s}$ \\
\hline $6 \mathrm{~s}$ & $7 \mathrm{~s}$ & $8 \mathrm{~s}$ & $9 \mathrm{~s}$ & $10 \mathrm{~s}$ & $11 \mathrm{~s}$ & $12 \mathrm{~s}$ & $13 \mathrm{~s}$ & $14 \mathrm{~s}$ & $15 \mathrm{~s}$ \\
\hline $16 \mathrm{~s}$ & $17 \mathrm{~s}$ & $18 \mathrm{~s}$ & $19 \mathrm{~s}$ & $20 \mathrm{~s}$ & $21 \mathrm{~s}$ & $22 \mathrm{~s}$ & $23 \mathrm{~s}$ & $24 \mathrm{~s}$ & $25 \mathrm{~s}$ \\
\hline
\end{tabular}

Para determinar os custos do manejo florestal, tornou-se necessário a subdivisão dos eventos que ocorrem no processo de produção. Segundo Mendes e Hildebrand (1997), esses eventos podem ser especificados de "processos", "atividades", "operações" e "recursos de produção", podendo ser representados da seguinte forma:

Processo de produção de toras $=\sum$ Atividades $=\sum$ Operações $=\sum$ Recursos consumidos

A atividade pode ser definida como sendo o conjunto de operações que demandam os recursos monetários e físicos para a produção de madeira, podendo ser classificada como componente do processo de produção. De acordo com Mendes (1998), há recursos que variam com a produção e há outros que não dependem do nível de produção. Assim, no curto prazo, há recursos fixos e variáveis, onde os fixos são os que não variam em função das alterações de produção, enquanto os recursos variáveis são os que, necessariamente, se alteram com as variações de produção, onde a natureza dessas duas categorias de recursos conduz aos custos fixos e variáveis.

\section{Custos fixos}

Os custos fixos incluem todas as formas de remuneração decorrentes da manutenção dos recursos fixos. Em razão da diferença de idade existente entre os vários bens móveis e imóveis, optou-se por utilizar o valor de aquisição desses bens. Os componentes dos custos fixos são:

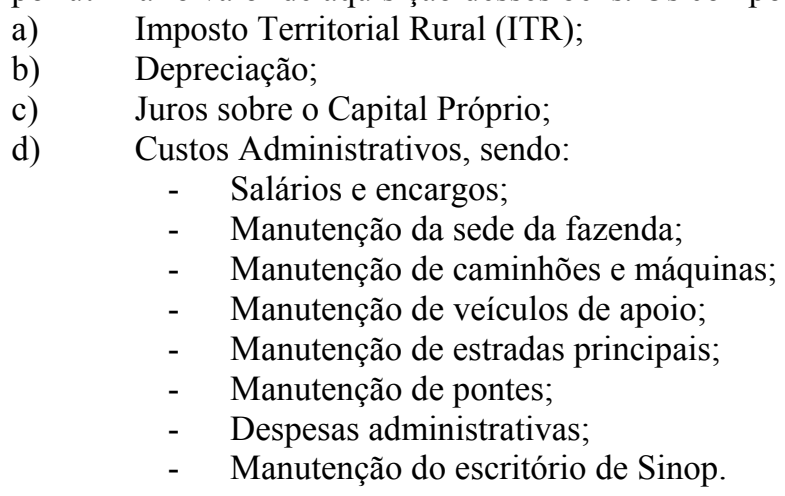


O Imposto Territorial Rural (ITR) incide sobre a propriedade de bens imóveis situados na zona rural. A depreciação econômica é um componente importante dos custos de produção, representando a perda do valor dos ativos, resultante do desgaste pelo uso, ação da natureza ou por obsoletismo técnico, e tem por objetivo apropriar o custo ou outro valor básico dos bens permanentes tangíveis, menos o valor residual (se houver), pela vida útil estimada do bem.

Segundo Timofeiczyk Júnior (2004), diversos métodos são utilizados para o cálculo da depreciação. Nesse trabalho optou-se pelo critério de depreciação linear, largamente utilizados na literatura econômica. A metodologia de cálculo para a obtenção desse item de custo se encontra abaixo:

onde: $\mathrm{De}=$ Depreciação $(\mathrm{R} \$ / \mathrm{ano})$;

$$
\mathrm{De}=(\text { V.A. }- \text { V.R. }) / \text { V.U. }
$$

$\mathrm{V} . \mathrm{A}=$ Valor de aquisição $(\mathrm{R} \$)$;

$\mathrm{V} . \mathrm{R}=$ Valor residual $(\mathrm{R} \$)$;

V.U = Vida útil (anos).

A remuneração do capital próprio representa o custo do valor perdido pela não aplicação na melhor alternativa econômica. De acordo com Hildebrand (1995), esse juro é calculado por meio da seguinte fórmula:

onde: $\mathrm{RC}=$ Remuneração do capital $(\mathrm{R} \$)$;

$$
\mathrm{RC}=\mathrm{VA} \cdot\left((1+\mathrm{i})^{\mathrm{n}} \cdot \mathrm{i}\right) /\left((1+\mathrm{i})^{\mathrm{n}}-1\right)-\mathrm{D}
$$

$\mathrm{VA}=$ Valor presente da aplicação ou aquisição (R\$);

$\mathrm{i} \quad=$ Taxa de juros $(\%)$;

$\mathrm{D}=$ Depreciação anual (R\$);

$\mathrm{n} \quad=$ Tempo (anos).

A taxa de juros utilizada para a remuneração do capital próprio é estimada em função das alternativas de aplicação ao alcance da empresa. Nesse estudo, a taxa de juros adotada para a determinação desse custo foi de $12 \%$ ao ano, baseada no trabalho de Timofeiczyk Júnior (2004). Os outros custos que não possuem vínculo direto com a produção foram alocados como custo administrativo.

\section{Custos variáveis}

Para a execução do Plano de Manejo Florestal Sustentável (PMFS) é necessária a elaboração do Plano Operacional Anual (POA), que deverá conter todas as atividades florestais da Empresa num determinado ano. O POA deve conter as seguintes atividades:

a) Atividade pré-exploratória;

b) Atividade exploratória;

c) Atividade pós-exploratória.

Tais atividades compõem os custos variáveis. Para a quantificação desses custos, foi considerado o gasto anual dessas atividades nos dois ciclos de corte analisados nessa pesquisa.

Atividade pré-exploratória

Para a obtenção do custo anual da atividade pré-exploratória, quantificou-se os custos das operações componentes dessa atividade. Tais operações são:

a) Elaboração do Plano de Manejo Florestal Sustentado (PMFS);

b) Censo florestal;

- Confecção de mapas;

- Inventário amostral;

- Demarcação das unidades de trabalho (UTs);

- Aberturas de picadas;

- Mensuração, avaliação e mapeamento das árvores;

c) Taxa do IBAMA (pré-exploratória);

d) Corte de cipós.

O censo florestal é uma operação importante, pois permite a localização, mensuração e avaliação das árvores de valor comercial, além de gerar informações úteis para o planejamento da exploração e das práticas silviculturais. A elaboração do PMFS e o censo florestal são realizados por terceiros com o 
pagamento efetuado por ha trabalhado. $\mathrm{O}$ valor gasto para a execução dessa operação foi fornecido pela Empresa que executa o manejo da floresta. Os custos da confecção de mapas, inventário amostral, demarcação das UTs, abertura de picadas, mensuração, avaliação e mapeamento das árvores foram obtidos pela Inflor - Engenharia Florestal, empresa que executa as operações acima citadas para empresas da região.

Os valores referentes às taxas a serem recolhidas ao IBAMA foram obtidos na sede desse órgão, no município de Sinop, MT. A taxa de vistoria prévia é realizada antes da aprovação do PMFS. Para o cálculo do valor dessa taxa, cobra-se um valor fixo até 250 ha, e sobre a área excedente, recolhe-se uma taxa sobre cada ha adicional. A operação de corte de cipós é realizada pela Empresa responsável pelo manejo, que repassou os custos por ha.

Atividade exploratória

Para a obtenção do custo anual da atividade exploratória, quantificou-se os custos de todas as operações componentes dessa atividade, sendo:

a) Taxas do IBAMA;

- Taxa de acompanhamento;

- $\quad$ Autorização de transporte de produtos florestais (ATPF);

b) Operações de exploração;

- Demarcação de estradas secundárias;

- Abertura de estradas secundárias;

- Demarcação de esplanada;

- Abertura de esplanada;

- Derrubada;

- Arraste;

- Traçamento;

- Cubagem;

c) Carregamento das toras nos caminhões.

A taxa de acompanhamento recolhida ao IBAMA é igual à taxa de vistoria prévia, já que ambas apresentam a mesma metodologia de cálculo. A Autorização de Transporte de Produtos Florestais (ATPF) é requisitada pela empresa por ocasião da aprovação do projeto de manejo. Cada ATPF consiste em uma carga de $35 \mathrm{~m}^{3}$.

As operações de exploração, que consistem na demarcação e abertura de estradas secundárias e esplanadas, derrubada, arraste, traçamento e cubagem, são realizadas por terceiros, com o pagamento sendo efetuado por metro cúbico esplanado. Para a determinação dos custos das operações de exploração, foram realizadas entrevistas com as empresas prestadoras desses serviços, chamadas de esplanadores, e com o gerente da Empresa contratante. O carregamento das toras nas carretas também é executado por terceiros, com o pagamento sendo efetivado por metro cúbico carregado.

\section{Atividade pós-exploratória}

Pouco se conhece sobre os métodos silviculturais da maioria das espécies encontradas nos trópicos, sendo um fator limitante e conflitante para se criar um modelo único de manejo florestal. As projeções sobre as reais necessidades da adoção de práticas silviculturais antes e após a exploração são baseadas em pesquisas científicas, requerendo confirmações por meio de experimentos de longo prazo. Os resultados até agora obtidos mostram que esses procedimentos têm uma influência positiva no crescimento da floresta remanescente.

Para a obtenção do custo anual da atividade pós-exploratória, quantificou-se os custos de todas as operações componentes dessa atividade, como sendo:

a) Tratos silviculturais;

- $\quad$ Corte de cipós;

- Favorecimento das espécies remanescentes;

b) Limpeza de estradas secundárias e esplanadas;

c) Proteção florestal;

d) Monitoramento e desenvolvimento da floresta. 
Os tratos silviculturais ocorrem apenas uma vez na UPA após a retirada da madeira. As operações de limpeza de estradas secundárias e esplanadas e o monitoramento do desenvolvimento da floresta são executados a cada cinco anos.

A introdução da limpeza de estradas secundárias e esplanadas na atividade pós-exploratória foi baseada no trabalho de Silva (1997), onde essa operação é realizada a cada cinco anos. De acordo com informações obtidas na Empresa, o intervalo de tempo ideal para executar a operação é de 5 anos. Intervalos menores não se justificam em razão da baixa densidade, e intervalos maiores podem comprometer a operação, diminuindo a produtividade e aumentando o custo.

Para executá-la, é necessário utilizar um trator Valmet 4 x 4 e uma roçadeira tracionada. Calculou-se o custo dessa operação com as seguintes informações:

a) Custo/hora do conjunto trator + roçadeira;

b) Turno de trabalho;

c) Eficiência;

d) Horas efetivas de trabalho por dia;

e) Produção (m/dia);

f) Densidade de estrada $(\mathrm{m} / \mathrm{ha})$.

A proteção florestal visa proteger a propriedade de invasões, incêndios florestais, caça e pesca ilegal e o meio ambiente em geral. Essa operação é realizada na área total da Fazenda, e os custos anuais estimados para a sua execução foram repassados pela Empresa.

Em estudos realizados por Arima e Barreto (2002), o corte de cipós foi realizado uma vez após a exploração, a fim de aumentar o crescimento das árvores de interesse comercial para o segundo corte. No presente estudo, assumiu-se que o corte de cipós foi realizado uma vez em cada UPA, cinco anos após a extração de madeira. Em razão da falta de informação a respeito do rendimento dessa operação, considerou-se o mesmo rendimento e custo verificado por ocasião da sua execução na atividade préexploratória.

A liberação da regeneração natural e o favorecimento de espécies remanescentes também são executados uma vez em cada UPA, um ano após a exploração. Entretanto, tais operações ainda não foram executadas pela Empresa, e, portanto, não há informações a respeito dos custos. Para a determinação desses, foram utilizadas as informações de dimensionamento de equipe, dias úteis de trabalho e produtividade contidos na pesquisa de Arima e Barreto (2002). De posse das informações, foram calculados os custos utilizando-se os salários e encargos pagos pela Empresa.

A introdução do monitoramento e desenvolvimento da floresta permite acompanhar o seu crescimento, a mortalidade, regeneração, bem como os danos causados pela exploração. O intervalo de tempo adotado para a execução foi baseado no trabalho do Silva (1997), que considerou intervalo de cinco anos entre as medições. O custo da realização deste trabalho foi obtido junto a Inflor - Engenharia Florestal.

\section{Impostos}

De acordo com Mendes e Hildebrand (1997), em função dos impostos serem indispensáveis no processo de produção, podem ser considerados como recursos, mesmo não gerando produção. Os mesmos autores citam que os impostos podem ser classificados como fixos ou variáveis.

Os impostos vinculados com a produção foram classificados como custo variável. De acordo com informações obtidas na Empresa, esses impostos são: Imposto de renda (IR), Contribuição Social sobre o Lucro Líquido (CSLL), Fundo de Assistência ao Trabalhador Rural (Funrural) e Instituto Nacional da Seguridade Social (INSS).

\section{Participação dos componentes de custos do manejo florestal}

Após o levantamento dos custos de produção, foram obtidos os seguintes resultados:

a) Quantificação monetária e em percentual dos custos fixos e variáveis totais que ocorreram no primeiro e segundo ciclos;

b) Quantificação monetária e em percentual dos componentes dos custos fixos e dos custos variáveis;

c) Quantificação monetária e em percentual das operações existentes nas atividades pré-exploratória, exploratória e pós-exploratória. 
Para a estimativa do volume disponível no segundo ciclo de corte, considerou-se que o crescimento das árvores aumentaria por meio da implantação dos tratos silviculturais. Amaral et al. (1998) afirmam que o acréscimo de volume a ser explorado no segundo ciclo, em função dos tratos silviculturais, é de $16 \%$. No caso da floresta, objeto desse estudo, a produção atual é de $12 \mathrm{~m}^{3} / \mathrm{ha}$, com a estimativa de colheita aumentando para $15 \mathrm{~m}^{3} /$ ha no segundo ciclo em função da implantação dos tratos silviculturais. No segundo ciclo de corte, para determinar volume comercializado por espécie, considerouse o mesmo percentual do primeiro ciclo.

\section{RESULTADOS}

\section{Custos fixos e custos variáveis}

Atualmente a empresa está fazendo a primeira intervenção nas Unidades de Produção Anual (UPAs). No atual nível de operação e comercialização, que é de $12 \mathrm{~m}^{3} /$ ha distribuídos em várias espécies, verificou-se que o custo médio de produção aumentou de $\mathrm{R} \$ 62,18 / \mathrm{m}^{3}$ no início de primeiro ciclo para $\mathrm{R} \$$ $65,71 / \mathrm{m}^{3}$ ao final desse. Essa elevação do custo médio de produção no primeiro ciclo ocorreu em função da implantação da atividade pós-exploratória nas Unidades de Produção Anual (UPAs), ocasionando aumento nos custos variáveis.

O custo variável foi responsável por $63,6 \%$ dos custos totais no início do primeiro ciclo de corte. A sua participação aumentou para $65,6 \%$ ao final desse, enquanto o custo fixo reduziu de $36,4 \%$ para $34,4 \%$ no final do ciclo (Figura 2). Em termos monetários, o custo variável por metro cúbico elevou-se de $\mathrm{R} \$ 39,56$ para $\mathrm{R} \$ 43,09$ no decorrer do primeiro ciclo, enquanto o custo fixo permaneceu em $\mathrm{R} \$ 22,62$ (Figura 3).

A partir do segundo ciclo o custo médio por metro cúbico foi reduzido de $\mathrm{R} \$ 65,71$ para $\mathrm{R} \$ 50,32$, representando uma queda de $23,42 \%$. A maior redução ocorreu nos custos variáveis, que passaram de $\mathrm{R} \$$ 43,09 para $R$ \$ 32,22, representando uma redução de 25,23\% (Figura 3). Isso ocorreu em função de algumas operações das atividades pré-exploratória e exploratória que deixaram de ser executadas, como o inventário amostral, demarcação das UTs, e abertura de estradas secundárias e esplanadas. Já o custo fixo passou de $\mathrm{R} \$ 22,62 / \mathrm{m}^{3}$ no primeiro ciclo de corte para $\mathrm{R} \$ 18,10 / \mathrm{m}^{3}$ no segundo ciclo, representando redução de $18,54 \%$, ocasionado pelo aumento do volume de $12 \mathrm{~m}^{3} /$ ha para $15 \mathrm{~m}^{3} /$ ha (Figura 3 ).

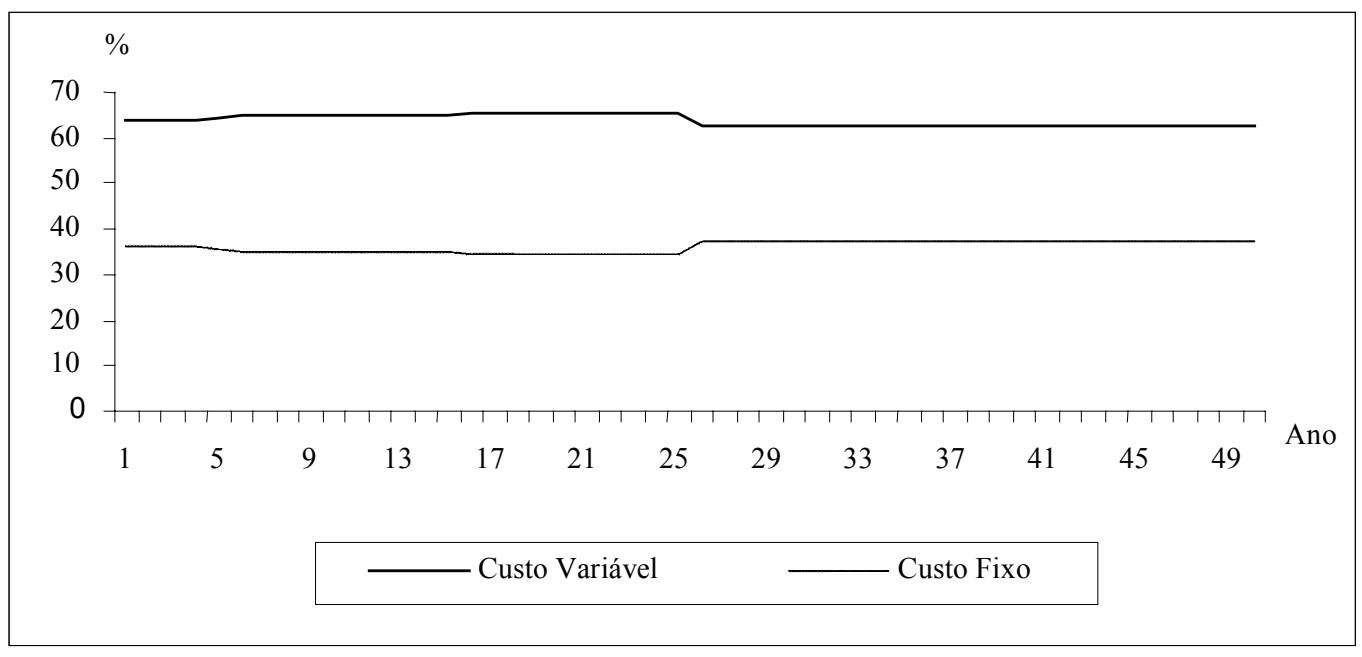

Figura 2. Participação percentual dos custos fixos e variáveis no custo total nos dois ciclos de corte. Figure 2. Percentage participation of fixed and variables costs in the total cost for two cutting cicles. 


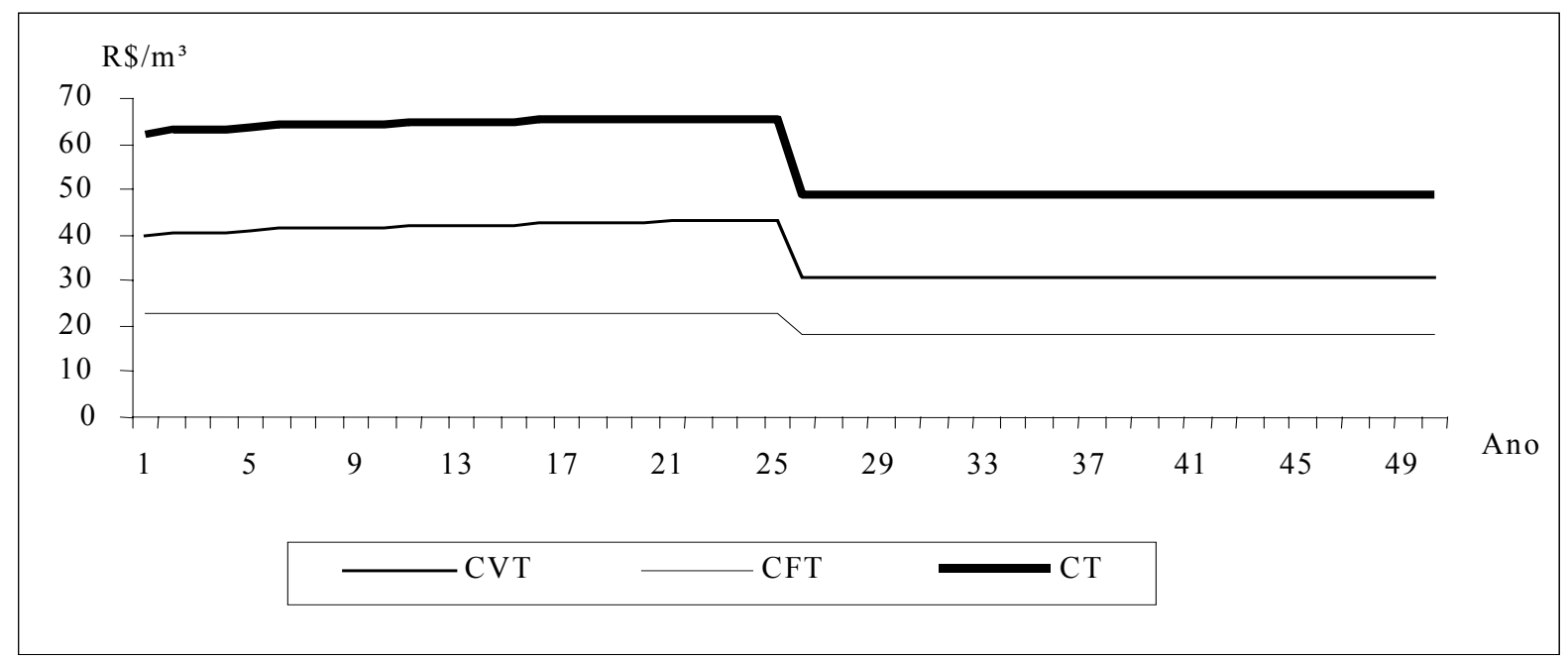

Figura 3: Custo total médio, custo fixo médio e custo variável médio por metro cúbico nos dois ciclos de corte $\left(\mathrm{R} \$ / \mathrm{m}^{3}\right)$.

Figure 3: Average total cost, average fixed cost and average varible cost per cubic meter for two cutting cicles $\left(\mathrm{R} \$ / \mathrm{m}^{3}\right)$.

Na Tabela 1 nota-se a alta participação do custo administrativo no custo fixo da empresa, e conseqüentemente no custo total. No primeiro ciclo, esses custos foram de $\mathrm{R} \$ 16,27 / \mathrm{m}^{3}$, representando $71,9 \%$ do custo fixo total. A depreciação da infra-estrutura, máquinas e equipamentos, juro sobre o capital investido e o Imposto Territorial Rural (ITR) oneraram a madeira em R $\$ 6,35 / \mathrm{m}^{3}$, representando $28,1 \%$ do custo fixo total.

É possível verificar que o custo fixo e seus componentes diminuíram a participação percentual no final do primeiro ciclo de corte, apesar de monetariamente não terem sofrido alterações. A implantação da atividade pós-exploratória no decorrer do primeiro ciclo promoveu o aumento dos custos variáveis, e consequentemente do custo total de produção, ocasionando essa diminuição percentual dos custos fixos. O custo administrativo reduziu a sua participação no custo total de $26,2 \%$ para $24,7 \%$ no final do primeiro ciclo, porém o custo por unidade de produto permaneceu em $\mathrm{R} \$ 16,27 / \mathrm{m}^{3}$ (Tabela 1).

Tabela 1. Custo fixo unitário no primeiro ciclo de corte $\left(\mathrm{R} \$ / \mathrm{m}^{3}\right)$.

Table 1. Unit fixed cost in the first cutting cicle $\left(\mathrm{R} \$ / \mathrm{m}^{3}\right)$.

\begin{tabular}{lcccc}
\hline \multirow{2}{*}{ ITENS DE CUSTOS } & \multirow{2}{*}{$\begin{array}{c}\text { CUSTO } \\
\left.\text { (R } \$ \mathrm{~m}^{3}\right)\end{array}$} & \% DO CUSTO & \multicolumn{2}{c}{ \% DO CUSTO TOTAL } \\
\cline { 4 - 5 } Custo Administrativo & 16,27 & 71,9 & 26,2 & 24,7 \\
Depreciação & 3,01 & 13,3 & 4,8 & 4,6 \\
Juros & 2,41 & 10,7 & 3,9 & 3,7 \\
ITR & 0,93 & 4,1 & 1,5 & 1,4 \\
\hline TOTAL & 22,62 & 100,0 & 36,4 & 34,4 \\
\hline
\end{tabular}

No segundo ciclo, houve decréscimo no custo fixo unitário para $\mathrm{R} \$ 18,10 / \mathrm{m}^{3}$, em razão do aumento do volume comercializado de $12 \mathrm{~m}^{3} /$ ha para $15 \mathrm{~m}^{3} /$ ha (Tabela 2). Entretanto, a participação percentual do custo fixo em relação ao custo total aumentou para $36 \%$, mantendo-se constante ao longo desse ciclo. Apesar do aumento da produção ter diluído os custos fixos por uma quantidade maior de 
produto, proporcionalmente essa redução foi menor que a respectiva redução nos custos variáveis, já que algumas operações das atividades pré-exploratória e exploratória deixaram de ser executadas no segundo ciclo.

Tabela 2. Custo fixo unitário no segundo ciclo de corte $\left(\mathrm{R} \$ / \mathrm{m}^{3}\right)$.

Table 2. Unit fixed cost in the second cutting cicle $\left(\mathrm{R} \$ / \mathrm{m}^{3}\right)$.

\begin{tabular}{lccc}
\hline ITENS DE CUSTOS & CUSTO $\left(\mathrm{R} \$ \mathrm{~m}^{3}\right)$ & \% DO CUSTO FIXO & \% DO CUSTO TOTAL \\
\hline Custo Administrativo & 13,01 & 71,9 & 25,9 \\
Depreciação & 2,41 & 13,3 & 4,8 \\
Juros & 1,92 & 10,7 & 3,8 \\
ITR & 0,76 & 4,1 & 1,5 \\
\hline TOTAL & 18,10 & 100,0 & 36,0 \\
\hline
\end{tabular}

Dos componentes do custo administrativo, os salários e a manutenção das estradas principais apresentaram os maiores custos, tanto no primeiro como no segundo ciclo de corte (Tabela 3). No primeiro ciclo, esses dois componentes juntos responderam por $57,0 \%$ do custo fixo e $20,8 \%$ do custo total de produção. No segundo ciclo, em função do aumento da produção, os custos administrativos foram reduzidos em $20 \%$, passando para $\mathrm{R} \$ 13,02 / \mathrm{m}^{3}$. Apesar da diminuição dos custos administrativos no segundo ciclo, a sua participação percentual teve uma ligeira elevação decorrente da maior redução dos custos variáveis nesse ciclo.

\section{Análise dos custos variáveis}

Atividade pré-exploratória

A atividade pré-exploratória teve baixo impacto no custo total, sendo responsável por R \$ $5,34 / \mathrm{m}^{3}$ no primeiro ciclo de corte, correspondendo a $8,6 \%$ do custo total de produção, como pode ser observado na Tabela 4. Em função do aumento dos custos no primeiro ciclo de corte, resultante da implantação dos tratos silviculturais na atividade pós-exploratória, a participação dessa atividade no custo total foi reduzida para $8,1 \%$ ao final do referido ciclo.

Nota-se que o censo florestal foi a operação mais onerosa da atividade pré-exploratória, sendo responsável por $6,3 \%$ do custo total do manejo no primeiro ciclo. Consumiu $73 \%$ dos recursos destinados a essa atividade, que para o atual nível de exploração da empresa representou $\mathrm{R} \$ 3,91 / \mathrm{m}^{3}$, dos quais $\mathrm{R} \$$ $1,92 / \mathrm{m}^{3}$ referiu-se à avaliação e mapeamento das árvores (Tabela 4). 
Tabela 3. Quantificação dos itens do custo administrativo nos dois ciclos de corte e sua participação percentual no custo fixo total e custo total.

Table 3. Quantification of the administrative cost items in the two cutting cicles and it percentagem participation for total fixed cost and total cost.

\begin{tabular}{lccccc}
\hline \multirow{2}{*}{ CUSTOS ADMINISTRATIVOS } & \multicolumn{2}{c}{ PRIMEIRO CICLO } & \multicolumn{2}{c}{ SEGUNDO CICLO } \\
\cline { 2 - 6 } & $\mathrm{R} \$ \mathrm{~m}^{3}$ & $\begin{array}{c}\text { \% do custo } \\
\text { fixo }\end{array}$ & $\begin{array}{c}\text { \% do } \\
\text { custo total }\end{array}$ & $\begin{array}{c}\text { R } \$ \mathrm{~m}^{3} \\
\text { \% do custo } \\
\text { total }\end{array}$ \\
\hline Salários e encargos & 6,45 & 28,5 & 10,4 & 5,16 & 10,4 \\
Manutenção de Estradas Principais & 6,45 & 28,5 & 10,4 & 5,16 & 10,4 \\
Manutenção Caminhões e Máquinas & 1,29 & 5,7 & 2,1 & 1,03 & 2,0 \\
Manutenção da Sede da Fazenda & 0,86 & 3,8 & 1,4 & 0,69 & 1,4 \\
Manutenção de Veículos de Apoio & 0,57 & 2,5 & 0,9 & 0,45 & 0,8 \\
Manutenção de pontes & 0,35 & 1,5 & 0,6 & 0,29 & 0,5 \\
Deslocamentos ente Sinop e Fazenda & 0,21 & 0,9 & 0,3 & 0,17 & 0,3 \\
Despesas Administrativas & 0,09 & 0,5 & 0,1 & 0,07 & 0,1 \\
\hline TOTAL & 16,27 & 71,9 & 26,2 & 13,02 & 25,9 \\
\hline
\end{tabular}

Tabela 4. Participação das etapas da atividade pré-exploratória no custo total e unitário nos dois ciclos de corte.

Table 4. Participation of the phases of pre-exploratory activitiy in the total and unit cost for two cutting cicles.

\begin{tabular}{|c|c|c|c|c|}
\hline \multirow{2}{*}{ PRÉ-EXPLORATÓRIA } & \multicolumn{2}{|c|}{$\mathrm{R} \$ / \mathrm{m}^{3}$} & \multicolumn{2}{|c|}{$\%$ DO CUSTO TOTAL } \\
\hline & $1^{0}$ Ciclo & $2^{\underline{0}}$ Ciclo & $1^{0}$ Ciclo & $2^{0}$ Ciclo \\
\hline \multicolumn{5}{|l|}{ Elaboração do PMFS } \\
\hline Confecção de Mapas & 0,21 & & 0,4 & \\
\hline Inventário Amostral & 0,46 & & 0,7 & \\
\hline SUB TOTAL 1 & 0,67 & & 1,1 & \\
\hline \multicolumn{5}{|l|}{ Censo Florestal } \\
\hline Demarcação das unidades de trabalho & 0,31 & & 0,5 & \\
\hline Aberturas de Picadas & 1,69 & 1,35 & 2,7 & 2,7 \\
\hline Mensuração, avaliação e mapeamento das árvores & 1,92 & 1,53 & 3,1 & 3,0 \\
\hline SUB TOTAL 2 & 3,91 & 2,88 & 6,3 & 5,7 \\
\hline Taxa do IBAMA & 0,05 & 0,04 & 0,1 & 0,1 \\
\hline Corte de Cipós & 0,71 & 0,57 & 1,1 & 1,1 \\
\hline TOTAL & 5,34 & 3,49 & $8,6 \%$ & $6,9 \%$ \\
\hline
\end{tabular}

O custo do corte de cipós varia em função da sua densidade, porém a sua execução é importante em razão da redução de danos na floresta remanescente e diminuição dos riscos de acidentes, além de criar melhores condições para a regeneração nos espaços abertos. O custo dessa operação foi de R\$ $0,71 / \mathrm{m}^{3}$, representando $1,1 \%$ do custo unitário de produção no primeiro ciclo. A taxa de vistoria prévia do IBAMA, ao contrário da afirmação de muitos produtores, representou pouco no custo unitário de 
produção, com $\mathrm{R} \$ 0,05 / \mathrm{m}^{3}$, ou aproximadamente $0,1 \%$ do custo total no primeiro ciclo, e $0,9 \%$ do custo dessa atividade (Tabela 4).

Algumas operações da atividade pré-exploratória são implantadas nas UPAs por ocasião da primeira intervenção, sendo dispensáveis no segundo ciclo por já terem sido implantadas. Essas operações são a confecção de mapas, inventário amostral e demarcação do talhão (Tabela 4). A diminuição de etapas, aliado ao aumento do volume explorado por hectare, reduziu o custo dessa atividade em $34,64 \%$, passando de $\mathrm{R} \$ 5,34 / \mathrm{m}^{3}$ para $\mathrm{R} \$ 3,49 / \mathrm{m}^{3}$. A participação no custo total reduziu de $8,6 \%$ para $6,9 \%$.

\section{Atividade exploratória}

Essa atividade apresentou-se como a de maior custo de produção, com $\mathrm{R} \$ 22,33 / \mathrm{m}^{3}$ no primeiro ciclo (Tabela 5). Entretanto, a participação percentual dessa atividade no custo total reduziu de $35,9 \%$ para 34,0\% no decorrer do primeiro ciclo, em razão da implantação dos tratos silviculturais na atividade pós-exploratória. $\mathrm{Na}$ atividade exploratória, as operações de exploração, que compreendem a demarcação e abertura de estradas secundárias e esplanadas, derrubada, arraste, traçamento e cubagem, custou R $\$$ $19,00 / \mathrm{m}^{3}$, representando $85,2 \%$ do custo da atividade. Do total dos recursos destinados para a atividade exploratória, $57,8 \%$ foram consumidos pelas operações de abertura de estradas e arraste, que juntas

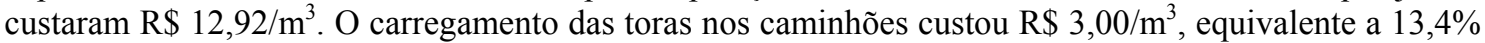
do custo da atividade exploratória.

Tabela 5. Participação das operações da atividade exploratória no custo unitário e total nos dois ciclos de corte.

Table 5. Participation of the exploratory activity operations in the total and unit cost for two cutting cicles.

\begin{tabular}{|c|c|c|c|c|}
\hline \multirow{2}{*}{ EXPLORATÓRIA } & \multicolumn{2}{|c|}{$\mathrm{R} \$ / \mathrm{m}^{3}$} & \multicolumn{2}{|c|}{$\%$ DO CUSTO TOTAL } \\
\hline & $1^{\mathrm{O}}$ Ciclo & $2^{\mathrm{O}}$ Ciclo & $1^{\mathrm{O}}$ Ciclo & $2^{\mathrm{O}}$ Ciclo \\
\hline \multicolumn{5}{|l|}{ Taxas do IBAMA } \\
\hline Taxa de Acompanhamento & 0,05 & 0,05 & 0,1 & 0,1 \\
\hline ATPF & 0,29 & 0,29 & 0,5 & 0,5 \\
\hline SUB TOTAL 4 & 0,33 & 0,34 & 0,6 & 0,6 \\
\hline \multicolumn{5}{|l|}{ Operações de exploração } \\
\hline Demarcação de estradas secundárias & 0,95 & & 1,5 & \\
\hline Abertura de estradas secundárias & 5,70 & & 9,2 & \\
\hline Demarcação de esplanada & 0,95 & & 1,5 & \\
\hline Abertura de esplanada & 0,95 & & 1,5 & \\
\hline Derrubada & 1,90 & 1,90 & 3,1 & 3,8 \\
\hline Arraste & 7,22 & 7,22 & 11,6 & 14,3 \\
\hline Traçamento & 0,95 & 0,95 & 1,5 & 1,9 \\
\hline Cubagem & 0,38 & 0,38 & 0,6 & 0,8 \\
\hline SUB TOTAL 5 & 19,00 & 10,45 & 30,5 & 20,8 \\
\hline CARREGAMENTO & 3,00 & 3,00 & 4,8 & 6,0 \\
\hline TOTAL & 22,33 & 13,79 & $35,9 \%$ & $27,4 \%$ \\
\hline
\end{tabular}

Entretanto, ao iniciar o segundo ciclo, a demarcação e abertura de estradas secundárias e esplanadas não foram executadas, haja vista já terem sido implementadas no primeiro ciclo. A não 
execução dessas operações ocasionou uma diminuição nos custos de exploração de $38,33 \%$, reduzindo o custo de $\mathrm{R} \$ 22,33 / \mathrm{m}^{3}$ para $\mathrm{R} \$ 13,79 / \mathrm{m}^{3}$ (Tabela 5 ). Ressalta-se que o cronograma da atividade pósexploratória contempla, a cada cinco anos, as manutenções das estradas secundárias e esplanadas.

Atividade pós-exploratória

No primeiro ciclo, o custo total do manejo aumentou devido às oscilações no cronograma da atividade pós-exploratória. Algumas operações dessa atividade ocorreram apenas uma vez na UPA após a intervenção na floresta. Entretanto, a limpeza de estradas secundárias e esplanadas, assim como o monitoramento e desenvolvimento da floresta ocorreram em intervalos de cinco anos, implicando na inserção de uma nova UPA no cronograma operacional dentro desse prazo.

Como são explorados $4.653,92$ ha por ano, tem-se que no $6^{0}$ ano foi realizada a limpeza das estradas secundárias e esplanadas e monitoramento da floresta, referente a primeira UPA trabalhada. Já no $7^{0}$ ano, tal trabalho foi realizado na segunda UPA, e assim sucessivamente. Dessa forma, do $6^{\underline{0}}$ ao $11^{\underline{0}}$ ano essas operações foram realizadas em $4.653,92$ ha anualmente. Do $12^{\underline{0}}$ ao $16^{\underline{0}}$ ano foram trabalhados $9.307,84$ ha, haja vista que as cinco primeiras UPAs sofreram a segunda intervenção, enquanto as últimas cinco tiveram a sua primeira intervenção, e assim sucessivamente.

Como é possível verificar na Figura 4, esse procedimento repetiu-se até o final do primeiro ciclo de corte (25 anos). Ao iniciar o segundo ciclo de corte, essas operações foram realizadas em 23.269,6 ha por ano, referente a cinco UPAs. Essa mudança no cronograma operacional afetou os custos à medida que mais unidades de produção são inseridas nas operações citadas.

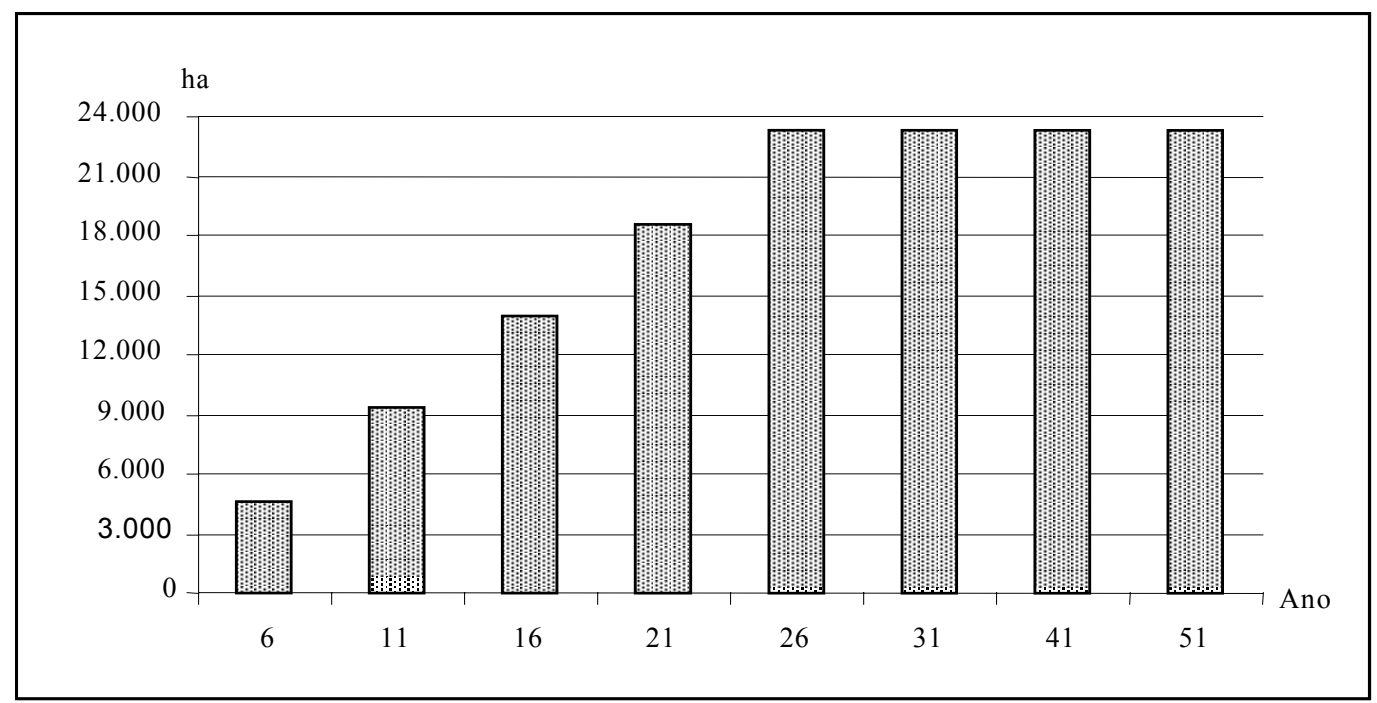

Figura 4. Aumento da área trabalhada para algumas operações da atividade pós-exploratória.

Figure 4. Increase of the worked area to some operations of the post-exploratory activity.

Conforme demonstrado na Tabela 6 , o custo da limpeza de estradas secundárias e esplanadas foi de $\mathrm{R} \$ 0,24 / \mathrm{m}^{3}$ do $6^{\circ}$ ao $11^{\circ}$ ano. Como houve inclusões de nova UPA a cada cinco anos no cronograma dessa operação, o custo aumentou $\mathrm{R} \$ 0,24 / \mathrm{m}^{3}$ dentro do referido prazo, atingindo o valor de $\mathrm{R} \$ 0,94 / \mathrm{m}^{3}$ no final do ciclo.

A partir do início do segundo ciclo, o custo ficou também em $\mathrm{R} \$ 0,94 / \mathrm{m}^{3}$, pois apesar de ter havido a inclusão de mais uma UPA no cronograma, não apresentou alterações em razão do aumento do volume explorado. No segundo ciclo não ocorreu mais alterações de custos em função de não ter havido introdução de novas UPAs no cronograma operacional (Tabela 6).

Sem a execução dessa operação, considerou-se que a reabertura das estradas secundárias e esplanadas tem custo aproximado de $70 \%$ dos custos da sua abertura, o que equivale a $\mathrm{R} \$ 4,66 / \mathrm{m}^{3}$. Com a introdução da limpeza de estradas secundárias e esplanadas, verificou-se que essa operação foi realizada 
cinco vezes em cada UPA no segundo ciclo, representando um custo de $\mathrm{R} \$ 0,94 / \mathrm{m}^{3}$, bem inferior ao custo de reabertura de estradas e esplanadas (Tabela 6).

A introdução e monitoramento da floresta teve o mesmo impacto no cronograma operacional apresentado na limpeza de estradas secundárias e esplanadas, custando $\mathrm{R} \$ 0,29 / \mathrm{m}^{3}$ do $6^{\underline{0}}$ ao $11^{\underline{0}}$ ano, e a partir do $12^{\underline{0}}$ ano aumentou $\mathrm{R} \$ 0,29$ a cada cinco anos. No segundo ciclo, o custo ficou em $\mathrm{R} \$ 1,16$, não sofrendo mais alterações de introdução de novas áreas no cronograma operacional (Tabela 6).

Em relação aos tratos silviculturais, o favorecimento das espécies remanescentes e a liberação da regeneração natural foram realizados apenas uma vez em cada UPA, ao custo de $\mathrm{R} \$ 0,36 / \mathrm{m}^{3}$ no primeiro ciclo. Como o pagamento dessa operação é realizado por hectare trabalhado, no segundo ciclo houve redução para $\mathrm{R} \$ 0,29 / \mathrm{m}^{3}$, em função do aumento da produção (Tabela 6 ).

O corte de cipós das árvores remanescentes também foi programado para ser realizado apenas uma vez em cada UPA, cinco anos após a extração. Essa etapa custou $\mathrm{R} \$ 0,71 / \mathrm{m}^{3}$ no primeiro ciclo de corte, e R $\$ 0,57$ no segundo ciclo. A proteção florestal é realizada na área total, ao custo de $\mathrm{R} \$ 0,89 / \mathrm{m}^{3}$. Entretanto, em função do aumento da produção no segundo ciclo, esse valor foi reduzido para $R \$ 0,71 / \mathrm{m}^{3}$ (Tabela 6).

Tabela 6. Comportamento dos custos da atividade pós-exploratória nos dois ciclos de corte $\left(\mathrm{R} \$ / \mathrm{m}^{3}\right)$. Table 6. Behavior of the costs of post-exploratory activity in the two cutting cycles $\left(\mathrm{R} \$ / \mathrm{m}^{3}\right)$.

\begin{tabular}{lccccccccc}
\hline & \multicolumn{10}{c}{ ANOS } \\
\cline { 2 - 8 } \multicolumn{1}{c}{ PÓS-EXPLORATÓRIA } & 1 & 2 & 6 & 11 & 16 & 21 & 26 & 31 \\
\hline $\begin{array}{l}\text { Limpeza de estradas e } \\
\text { esplanadas }\end{array}$ & & & 0,24 & 0,48 & 0,72 & 0,94 & 0,94 & 0,94 \\
$\begin{array}{l}\text { Monitoramento e } \\
\text { desenvolvimento }\end{array}$ & & & 0,29 & 0,58 & 0,87 & 1,16 & 1,16 & 1,16 \\
$\begin{array}{l}\text { Favorecimentos espécies } \\
\text { remanescentes }\end{array}$ & 0,36 & 0,36 & 0,36 & 0,36 & 0,36 & 0,29 & 0,29 \\
$\begin{array}{l}\text { Liberação da regeneração } \\
\text { natural }\end{array}$ & 0,36 & 0,36 & 0,36 & 0,36 & 0,36 & 0,29 & 0,29 \\
$\begin{array}{l}\text { Proteção florestal } \\
\text { Corte de cipós }\end{array}$ & 0,89 & 0,89 & 0,89 & 0,89 & 0,89 & 0,89 & 0,71 & 0,71 \\
\hline
\end{tabular}

A participação da atividade pós-exploratória no custo unitário total elevou-se de $1,4 \%$ no início do primeiro ciclo de corte para 6,7\% ao final desse (Tabela 7). No segundo ciclo, em função do aumento da produção, o custo unitário da atividade reduziu para $\mathrm{R} \$ 3,96 / \mathrm{m}^{3}$. Entretanto, a participação relativa no custo total elevou-se para $7,9 \%$ em razão da redução dos custos das atividades pré-exploratória e exploratória, aumentando a participação da atividade pós-exploratória no custo total.

Tabela 7. Evolução do custo da atividade pós-exploratória no custo total e unitário.

Table 7. Evolution of the cost of post-exploratory activity in the total and unit cost.

\begin{tabular}{ccc}
\hline ANO & $\mathrm{R} \$ \mathrm{~m}^{3}$ & \% DO CUSTO TOTAL \\
\hline 1 & 0,89 & 1,4 \\
2 & 1,61 & 2,5 \\
6 & 2,85 & 4,4 \\
11 & 3,38 & 5,2 \\
16 & 3,91 & 6,0 \\
21 & 4,42 & 6,7 \\
26 & 3,96 & 7,9 \\
51 & 3,96 & 7,9 \\
\hline
\end{tabular}




\section{Impostos}

Os impostos pagos pela empresa totalizaram $\mathrm{R} \$ 11,00 / \mathrm{m}^{3}$, valores esses repassados pelo escritório central da empresa em Sinop. No primeiro ciclo, os impostos foram responsáveis por $28 \%$ do custo variável médio e por $18 \%$ do custo total médio. Já no segundo ciclo, a participação dos impostos no custo variável médio aumentou para $34,2 \%$, representando $22 \%$ do custo médio de produção nesse ciclo.

\section{CONCLUSÕES}

O custo variável foi o principal componente do custo total, elevando-se no primeiro ciclo de corte em função da implantação da atividade pós-exploratória.

No segundo ciclo de corte, os custos variáveis apresentaram redução em razão do aumento de $25 \%$ na produtividade, aliado ao fato que várias operações das atividades pré-exploratória e exploratória que foram executadas no primeiro ciclo, não ocorreram a partir do segundo ciclo.

$\mathrm{Na}$ atividade exploratória ocorreu o maior custo do manejo no primeiro ciclo de corte. Das operações dessa atividade, a abertura de estradas secundárias e o arraste foram as que apresentaram os maiores custos.

Das atividades componentes do custo variável, a atividade pré-exploratória apresentou o menor impacto no custo total, elevando-se ao longo do primeiro ciclo, estabilizando-se a partir do segundo ciclo.

Ao contrário da afirmação de muitos madeireiros, a taxa de vistoria prévia recolhida ao IBAMA teve baixa participação no custo total de produção.

No segundo ciclo, a demarcação e abertura de estradas secundárias e esplanadas não foram executadas, tendo em vista já terem sido implementadas no primeiro ciclo, ocasionando uma diminuição nos custo de exploração.

A atividade pós-exploratória apresentou custos crescentes no primeiro ciclo, em função das operações que foram implementadas. No segundo ciclo, o custo total dessa atividade permaneceu estável, porém o custo por metro cúbico apresentou redução devido ao aumento da produtividade.

O custo fixo no primeiro ciclo ficou inalterado monetariamente, entretanto a sua participação percentual no custo total apresentou redução em razão do aumento do custo variável.

No segundo ciclo, o aumento da produção diluiu os custos fixos por uma quantidade maior de produto, porém esta redução foi menor que a respectiva redução nos custos variáveis, em função das operações das atividades pré-exploratória e exploratória que deixaram de ser executadas no segundo ciclo.

O custo administrativo foi o principal componente do custo fixo. Dos componentes do custo administrativo, os mais representativos foram os salários e encargos, e a manutenção de estradas principais.

\section{REFERÊNCIAS}

ACSELRAD, H. Sustentabilidade, discursos e disputas. In: WORKSHOP Sustentabilidade: Perspectivas Não-Governamentais, 1995, Rio de Janeiro. Anais... Rio de Janeiro: IBASE, 1995.

AMARAL, P.; VERÍSSIMO, A.; BARRETO, P.; VIDAL, E. Floresta para sempre: um manual para a produção de madeira na Amazônia. Belém: Imazon, 1998.

ARIMA, E.; BARRETO, P. Rentabilidade da produção de madeira em terras públicas e privadas na região de cinco florestas nacionais da Amazônia. Brasília: Ministério do Meio Ambiente, 2002. 49p.

ARIMA, E.; VERÍSSIMO, A. Brasil em ação: Ameaças e oportunidades econômicas na fronteira Amazônica. Série Amazônica n.19. Belém: Imazon, 2002. 24p.

CUNHA, U. S. Análise da estrutura espacial horizontal de uma floresta de terra firme da Amazônia. Curitiba, 2003, 126 f. Tese (Doutorado em Ciências Florestais) - Setor de Ciências Agrárias, Universidade Federal do Paraná. 
HILDEBRAND, E. Sistema de apropriação e análise de custos para a empresa florestal. Curitiba, 1995, 145 f. Dissertação (Mestrado em Ciências Florestais) - Setor de Ciências Agrárias, Universidade Federal do Paraná.

HUMMEL, A. C. Normas de acesso ao recurso florestal na Amazônia brasileira: O caso do manejo florestal madeireiro. Manaus, 2001, 83 f. Dissertação (Mestrado em Ciências de Florestas Tropicais) INPA/UA.

MENDES, J. T. G. Economia Agrícola - Princípios Básicos e Aplicações. Curitiba: ZNT Ltda. 2 ed, 1998.

MENDES, J. B.; HILDEBRAND, E. Procedimentos para a concepção de um sistema de custos florestais. Curitiba: Fupef - Silviconsult Engenharia, p. 1-26, 1997.

SILVA, Z. A. G. P. Análisis econômico del manejo forestal em el Estado de Acre, Brasil. Simpósio Internacional; Posibilidades de Manejo Forestal Sostenible em América Tropical. Santa Cruz de la Sierra, Bolívia, 15-20 Julio 1997. 11 p.

SOUZA, A. L. P. Desenvolvimento sustentável, manejo florestal e o uso dos recursos madeireiros na Amazônia: desafios, possibilidades e limites. Belém: UFPA/NAEA, 2002. 255 p.

TIMOFEICZYK, R. Jr. Análise econômica do manejo de baixo impacto em florestas tropicais - um estudo de caso. Curitiba, 2004, 126 f. Tese (Doutorado em Engenharia Florestal) - Setor de Ciências Agrárias, Universidade Federal do Paraná.

ZACHOW, R. Metodologia para Monitoramento de Projetos de Manejo em Florestas Naturais Tropicais Baseadas em Critérios Normativos. Curitiba, 1999, 242 f.Tese (Doutorado em Ciências Florestais) - Setor de Ciências Agrárias, Universidade Federal do Paraná. 